PROCEEDINGS OF THE

AMERICAN MATHEMATICAL SOCIETY

Volume 132, Number 10, Pages 2969-2972

S 0002-9939(04)07462-3

Article electronically published on June 2, 2004

\title{
A NEW PROOF FOR ROCKAFELLAR'S CHARACTERIZATION OF MAXIMAL MONOTONE OPERATORS
}

\author{
S. SIMONS AND C. ZĂLINESCU
}

(Communicated by Jonathan M. Borwein)

\begin{abstract}
We provide a new and short proof for Rockafellar's characterization of maximal monotone operators in reflexive Banach spaces based on $\mathrm{S}$. Fitzpatrick's function and a technique used by R. S. Burachik and B. F. Svaiter for proving their result on the representation of a maximal monotone operator by convex functions.
\end{abstract}

\section{The REsult}

Throughout this note $(X,\|\cdot\|)$ is a reflexive Banach space and $X^{*}$ is its topological dual space whose dual norm is denoted by $\|\cdot\|_{*}$. Then the topological dual of $X \times X^{*}$ is $X^{*} \times X$, the pairing being given by $\left\langle\left(x, x^{*}\right),\left(u^{*}, u\right)\right\rangle:=\left\langle x, u^{*}\right\rangle+\left\langle u, x^{*}\right\rangle$, where, as usual, $\left\langle x, u^{*}\right\rangle:=u^{*}(x)$ for $x \in X$ and $u^{*} \in X^{*}$. Let $A: X \rightrightarrows X^{*}$ be a multivalued operator (or multifunction) whose graph gr $A:=\left\{\left(x, x^{*}\right) \mid x^{*} \in A(x)\right\}$ is nonempty. Recall that $A$ is monotone if

$$
\left\langle x-y, x^{*}-y^{*}\right\rangle \geq 0 \quad \forall\left(x, x^{*}\right),\left(y, y^{*}\right) \in \operatorname{gr} A ;
$$

$A$ is maximal monotone if $A$ is monotone and for any monotone operator $B: X \rightrightarrows$ $X^{*}$ with $\operatorname{gr} A \subset \operatorname{gr} B$ we have that $A=B$.

It is well known (and obvious) that if $A: X \rightrightarrows X^{*}$ is a maximal monotone operator and $\left(y, y^{*}\right) \in X \times X^{*}$, then

$$
\inf _{\left(a, a^{*}\right) \in \operatorname{gr} A}\left\langle a-y, a^{*}-y^{*}\right\rangle \leq 0, \text { with equality } \Leftrightarrow\left(y, y^{*}\right) \in \operatorname{gr} A .
$$

Define

$$
g: X \times X^{*} \rightarrow \overline{\mathbb{R}}, \quad g\left(y, y^{*}\right):=\sup _{\left(a, a^{*}\right) \in \operatorname{gr} A}\left[\left\langle a, y^{*}\right\rangle+\left\langle y, a^{*}\right\rangle-\left\langle a, a^{*}\right\rangle\right] .
$$

Then (1.1) can be written as

$$
\forall\left(y, y^{*}\right) \in X \times X^{*},\left\langle y, y^{*}\right\rangle \leq g\left(y, y^{*}\right), \text { with equality } \Leftrightarrow\left(y, y^{*}\right) \in \operatorname{gr} A .
$$

As Fitzpatrick [2] observed, $g$ is a lower semicontinuous proper convex function when $A$ is maximal monotone. In the next statement the subdifferential of $g$ is meant in the sense of convex analysis; in particular, the subdifferential is empty at a point outside the domain of the function.

Received by the editors February 6, 2003.

2000 Mathematics Subject Classification. Primary 47H05; Secondary $26 \mathrm{~B} 25$.

Key words and phrases. Maximal monotone operator, convex function, duality mapping. 
Lemma 1.1. Assume that $A$ is maximal monotone and that $\left(u^{*}, u\right) \in \partial g\left(v, v^{*}\right)$. Then $\left\langle v-u, v^{*}-u^{*}\right\rangle \leq 0$. Moreover, if $\left\langle v-u, v^{*}-u^{*}\right\rangle=0$, then $\left(u, u^{*}\right) \in \operatorname{gr} A$.

Proof. From (1.3) we have that

$$
\begin{aligned}
\left\langle v-u, v^{*}-u^{*}\right\rangle & =\left\langle v, v^{*}\right\rangle-\left\langle v, u^{*}\right\rangle-\left\langle u, v^{*}\right\rangle+\left\langle u, u^{*}\right\rangle \\
& \leq g\left(v, v^{*}\right)-\left\langle v, u^{*}\right\rangle-\left\langle u, v^{*}\right\rangle+\left\langle u, u^{*}\right\rangle .
\end{aligned}
$$

Fix $\left(a, a^{*}\right) \in \operatorname{gr} A$. Since $\left(u^{*}, u\right) \in \partial g\left(v, v^{*}\right)$ and using (1.3) again, we have that

$g\left(v, v^{*}\right) \leq g\left(a, a^{*}\right)-\left\langle\left(a, a^{*}\right)-\left(v, v^{*}\right),\left(u^{*}, u\right)\right\rangle=\left\langle a, a^{*}\right\rangle+\left\langle v-a, u^{*}\right\rangle+\left\langle u, v^{*}-a^{*}\right\rangle$.

Therefore,

$$
\left\langle v-u, v^{*}-u^{*}\right\rangle \leq\left\langle a, a^{*}\right\rangle-\left\langle a, u^{*}\right\rangle-\left\langle u, a^{*}\right\rangle+\left\langle u, u^{*}\right\rangle=\left\langle a-u, a^{*}-u^{*}\right\rangle .
$$

Hence

$$
\left\langle v-u, v^{*}-u^{*}\right\rangle \leq \inf _{\left(a, a^{*}\right) \in \operatorname{gr} A}\left\langle a-u, a^{*}-u^{*}\right\rangle .
$$

The first part of the result follows from (1.1). If $\left\langle v-u, v^{*}-u^{*}\right\rangle=0$, then (1.4) gives $\inf _{\left(a, a^{*}\right) \in \operatorname{gr} A}\left\langle a-u, a^{*}-u^{*}\right\rangle \geq 0$, and so, using again (1.1), we obtain that $\left(u, u^{*}\right) \in \operatorname{gr} A$.

Recall that the duality mapping of $X$ is the multifunction $J_{X}: X \rightrightarrows X^{*}$ defined by $J_{X}(x):=\left\{x^{*} \in X^{*} \mid\left\langle x, x^{*}\right\rangle=\|x\|^{2}=\left\|x^{*}\right\|_{*}^{2}\right\}=\partial\left(\frac{1}{2}\|\cdot\|^{2}\right)$; then $J_{X}^{-1}\left(x^{*}\right)=\partial\left(\frac{1}{2}\|\cdot\|_{*}^{2}\right)$. Using Lemma 1.1] we get the generalization in [7, Thm. 10.7] of Rockafellar's celebrated characterization of maximal monotone operators.

Theorem 1.2. Let $X$ be a reflexive Banach space, and let $A: X \rightrightarrows X^{*}$ be monotone. Then $A$ is maximal monotone if and only if $\operatorname{gr} A+\operatorname{gr}\left(-J_{X}\right)=X \times X^{*}$.

Proof. The proof of the sufficiency does not suppose the reflexivity of the space and is well known. Indeed, let $\left(y, y^{*}\right) \in X \times X^{*}$ be such that $\left\langle a-y, a^{*}-y^{*}\right\rangle \geq 0$ for every $\left(a, a^{*}\right) \in \operatorname{gr} A$. By hypothesis there exist $\left(a, a^{*}\right) \in \operatorname{gr} A$ and $\left(u, u^{*}\right) \in \operatorname{gr}\left(-J_{X}\right)$ such that $\left(y, y^{*}\right)=\left(a, a^{*}\right)+\left(u, u^{*}\right)$. Then

$$
0 \leq\left\langle a-y, a^{*}-y^{*}\right\rangle=\left\langle-u,-u^{*}\right\rangle=-\left\langle u,-u^{*}\right\rangle=-\|u\|^{2}=-\left\|-u^{*}\right\|_{*}^{2},
$$

and so $u=0$ and $u^{*}=0$ (we have used the fact that $-u^{*} \in J_{X}(u)$ ). It follows that $\left(y, y^{*}\right)=\left(a, a^{*}\right) \in \operatorname{gr} A$. Hence $A$ is maximal monotone.

Assume that $A$ is maximal monotone. Let us prove that $(0,0) \in \operatorname{gr} A+\operatorname{gr}\left(-J_{X}\right)$.

Consider $g$ defined in (1.2); as observed above, $g$ is a proper lower semicontinuous convex function. Consider the function $h: X \times X^{*} \rightarrow \overline{\mathbb{R}}$ defined by

$$
h\left(x, x^{*}\right):=\frac{1}{2}\|x\|^{2}+\frac{1}{2}\left\|x^{*}\right\|_{*}^{2}+g\left(x, x^{*}\right) .
$$

It is obvious that $h$ is proper, lower semicontinuous, (strongly) coercive and convex. The space $X \times X^{*}$ being reflexive, there exists $\left(v, v^{*}\right)$ minimizing $h$ on $X \times X^{*}$. Hence $(0,0) \in \partial h\left(v, v^{*}\right)$. Moreover, because $h$ is the sum of three convex functions, two of them being continuous, one obtains that

$$
(0,0) \in J_{X}(v) \times J_{X}^{-1}\left(v^{*}\right)+\partial g\left(v, v^{*}\right),
$$

and so there exists $\left(u^{*}, u\right) \in \partial g\left(v, v^{*}\right)$ such that $-u^{*} \in J_{X}(v)$ and $-u \in J_{X}^{-1}\left(v^{*}\right)$ (equivalently $-v^{*} \in J_{X}(u)$ ). Using Lemma 1.1 we obtain that $\left\langle v-u, v^{*}-u^{*}\right\rangle \leq 0$. 
Because $-u^{*} \in J_{X}(v)$ and $-v^{*} \in J_{X}(u)$ we have that $\left\langle v,-u^{*}\right\rangle=\|v\|^{2}=\left\|u^{*}\right\|_{*}^{2}$, $\left\langle u,-v^{*}\right\rangle=\|u\|^{2}=\left\|v^{*}\right\|_{*}^{2}$, and so

$$
\begin{aligned}
0 & \geq\left\langle v-u, v^{*}-u^{*}\right\rangle=\left\langle v, v^{*}\right\rangle+\left\langle v,-u^{*}\right\rangle+\left\langle u,-v^{*}\right\rangle+\left\langle u, u^{*}\right\rangle \\
& \geq\|v\|^{2}-2\|v\| \cdot\|u\|+\|u\|^{2}=(\|v\|-\|u\|)^{2} \geq 0 .
\end{aligned}
$$

Hence each inequality in (1.5) is in fact an equality. Therefore $\left\langle v-u, v^{*}-u^{*}\right\rangle=0$ and $\|v\|=\|u\|\left(=\left\|v^{*}\right\|_{*}=\left\|u^{*}\right\|_{*}\right),\left\langle-u, u^{*}\right\rangle=\|v\| \cdot\|u\|=\|-u\|^{2}=\left\|u^{*}\right\|_{*}^{2}$; thus $u^{*} \in J_{X}(-u)$. Using the last part of Lemma 1.1 we obtain that $\left(u, u^{*}\right) \in \operatorname{gr} A$. Because $\left(-u,-u^{*}\right) \in \operatorname{gr}\left(-J_{X}\right)$, we deduce that $(0,0)=\left(u, u^{*}\right)+\left(-u,-u^{*}\right) \in$ $\operatorname{gr} A+\operatorname{gr}\left(-J_{X}\right)$.

Let $\left(y, y^{*}\right) \in X \times X^{*}$ be fixed. Taking $A^{\prime}: X \rightrightarrows X^{*}$ with $\operatorname{gr} A^{\prime}=\operatorname{gr} A-\left(y, y^{*}\right)$, $A^{\prime}$ is maximal monotone. By what precedes we obtain that $(0,0) \in \operatorname{gr} A^{\prime}+\operatorname{gr}\left(-J_{X}\right)$; that is, $\left(y, y^{*}\right) \in \operatorname{gr} A+\operatorname{gr}\left(-J_{X}\right)$. The conclusion follows.

The proof of this theorem in [7. Thm. 10.6] is based on several results, some of them using minimax theorems (though it is now known that the minimax theorems can be replaced by an appropriately generalized form of the Hahn-Banach theorem). When $J_{X}$ and $J_{X}^{-1}$ are single-valued, the preceding result yields (easily) Rockafellar's characterization of maximal monotonicity of $A$ in [6] (see also [7] Thm. 10.7, Rem. 10.8]):

Theorem 1.3. Let $X$ be a reflexive Banach space, and let $A: X \rightrightarrows X^{*}$ be monotone. If $A$ is maximal monotone, then $A+J_{X}$ is onto. Conversely, if $A+J_{X}$ is onto and $J_{X}, J_{X}^{-1}$ are single-valued, then $A$ is maximal monotone.

Without asking that $J_{X}$ and $J_{X}^{-1}$ be single-valued in the last part of the preceding theorem, the statement is not true, as can be seen from [7, page 39].

Our proof of Theorem 1.2 is inspired by the proof of [1, Thm. 3.1], where a similar function to $h$ (above) is considered. In order to prove their result, Burachik and Svaiter renorm the space $X$ and apply Rockafellar's theorem; applying Theorem 1.2, there is no need to renorm the space.

It is possible to prove Theorem 1.2 using a characterization of maximal monotone subsets of $X \times X^{*}$ given by Martinez-Legaz and Théra in [4] and some properties of convex functions associated to monotone subsets from Penot [5], but the use of Lemma 1.1 leads to a much shorter proof.

Without asking $X$ to be reflexive, the statement of Theorem 1.2 does not hold. Indeed, taking $A: X \rightrightarrows X^{*}$ defined by $A(x):=\{0\}, A$ is maximal monotone and $\operatorname{gr} A+\operatorname{gr}\left(-J_{X}\right)=X \times \operatorname{Im} J_{X}$. But, for $X$ a Banach space, $J_{X}$ is onto if and only if $X$ is reflexive.

If $X$ is a nonreflexive Banach space, we write ${ }^{\wedge}$ for the canonical map from $X$ into its bidual $X^{* *}$. Now let $A: X \rightrightarrows X^{*}$ be maximal monotone. We say that $A$ is of type (NI) if, whenever $\left(y^{* *}, y^{*}\right) \in X^{* *} \times X^{*}$,

$$
\inf _{\left(a, a^{*}\right) \in \operatorname{gr} A}\left\langle a^{*}-y^{*}, \widehat{a}-y^{* *}\right\rangle \leq 0 .
$$

Furthermore, in [3], Gossez introduced the multifunction $\bar{A}: X^{* *} \rightrightarrows X^{*}$ defined by:

$$
y^{*} \in \bar{A} y^{* *} \quad \Longleftrightarrow \quad \inf _{\left(a, a^{*}\right) \in \operatorname{gr} A}\left\langle a^{*}-y^{*}, \widehat{a}-y^{* *}\right\rangle \geq 0 .
$$

The following result was proved in [7, Lemma 27.5], and was used in [7] to give a precise description of the closure of the range of a maximal monotone operator of 
Gossez's "type (D)", and in [8] to prove that certain maximal monotone multifunctions have an approximate "Brøndsted-Rockafellar" property. Let $A: X \rightrightarrows X^{*}$ be maximal monotone of type (NI). Then there exist $u^{* *} \in X^{* *}$ and $u^{*} \in \bar{A} u^{* *}$ such that $u^{* *} \in-J_{X^{*}}\left(u^{*}\right)$. It would be very nice if the techniques of this paper could be used to prove this result, but we do not know if this is the case.

\section{REFERENCES}

[1] R. S. Burachik and B. F. Svaiter, Maximal monotonicity, conjugation and the duality product, Proc. Amer. Math. Soc. 131 (2003), 2379-2383. MR 2004a:49037

[2] S. Fitzpatrick, Representing monotone operators by convex functions, Workshop/Miniconference on Functional Analysis and Optimization (Canberra, 1988), Austral. Nat. Univ., Canberra, 1988, pp. 59-65.

[3] J.-P. Gossez, Opérateurs monotones non linéaires dans les espaces de Banach non réflexifs J. Math. Anal. Appl. 34 (1971), 371-395. MR 47:2442

[4] J. E. Martinez-Legaz and M. Théra, A convex representation of maximal monotone operators, J. Nonlinear Convex Anal. 2 (2001), 243-247. MR 2002e:49035

[5] J.-P. Penot, The relevance of convex analysis for the study of monotonicity, Tech. report, University of Pau, Pau, 2002.

[6] R. T. Rockafellar, On the maximality of sums of nonlinear monotone operators, Trans. Amer. Math. Soc. 149 (1970), 75-88. MR 43:7984

[7] S. Simons, Minimax and monotonicity, Springer-Verlag, Berlin, 1998. MR 2001h:49002

[8] S. Simons, Maximal monotone multifunctions of Brøndsted-Rockafellar type, Set-Valued Anal. 7 (1999), 255-294. MR 2001b:47100

Department of Mathematics, University of California, Santa Barbara, California 93106

E-mail address: simons@math.ucsb.edu

Faculty of Mathematics, University "Al. I. Cuza" iaşi, Bd. Carol I, Nr. 11, 700506 IAŞI, ROMANIA

E-mail address: zalinesc@uaic.ro 